\title{
Laser Desorption Ion Trap Mass Spectrometry of Self-Assembled Monolayers
}

\author{
John A. Burroughs and Luke Hanley \\ Department of Chemistry, University of Illinois at Chicago, Chicago, Illinois, USA
}

\begin{abstract}
It is demonstrated that laser desorption ion trap mass spectrometry (LD-ITMS) can be successfully applied to the chemical analysis of a monolayer of adsorbates on a solid surface. Negative ion spectra obtained from LD-ITMS of self-assembled monolayers adsorbed from solutions of alkanethiols $\left(\mathrm{CH}_{3}\left(\mathrm{CH}_{2}\right)_{n} \mathrm{SH}\right.$ with $\mathrm{n}=5,9$, and 15) unto polycrystalline gold surfaces displayed clear ion peaks corresponding to the sulfonate adsorbate species. Sulfonate ions with the general formula $\mathrm{CH}_{3}\left(\mathrm{CH}_{2}\right)_{n} \mathrm{SO}_{3}$ were detected at $\mathrm{m} / \mathrm{z} 165,221$, and 305 , respectively, and were derived from the partial oxidation of the corresponding alkanethiol self-assembled monolayers. Little fragmentation and no clustering was observed in these mass spectra. These results indicate that the sensitivity of LD-ITMS is sufficient to allow its application to a wide array of problems in surface science. (J Am Soc Mass Spectrom $1993,4,968-970$ )
\end{abstract}

\section{$\mathrm{L}$} aser desorption mass spectrometric analysis of monolayer coverages of adsorbates has been previously achieved with quadrupole, time-of-flight, and Fourier transform ion cyclotron resonance instruments $[1,2]$ (see ref 1 for a series of articles on surface analysis via laser desorption mass spectrometry). The former two instruments have the advantage of experimental simplicity, whereas the latter allows tandem and higher orcter mass spectrometric analyses (MS'), which are of particular utility in the chemical identification of complex organic surfaces. However, the high magnetic fields associated with ion cyclotron resonance instruments interfere with the electron spectrometers typically present in multiprobe surface analysis systems.

Ion trap mass spectrometers should be ideal for integration into surface analysis systems given their lack of magnetic fields, small size, high mass range, $\mathrm{MS}^{\mathrm{n}}$ capability, high sensitivity, and potentially high mass resolution [3]. Ion trap mass spectrometers have been used in a variety of configurations [3], including many with ion or laser desorption for the analysis of solids or thick films [4-9]. We show here that laser desorption ion trap mass spectrometry (LD-ITMS) can also be successfully applied to the chemical analysis of a monolayer of adsorbates on a solid surface.

\section{Experimental}

An ion trap mass spectrometer was constructed by removing the electrodes from a commercially available

Address reprint requests to Luke Hanlcy, Department of Chemintry (M/C 111), University of Illinois at Chicago, 845 West Taylor Street, Chicago, IL 60607-7061. ion trap detector (ITD 700, Finnigan MAT, San Jose, CA) and mounting them inside a diffusion-pumped vacuum chamber. A $6 \mathrm{kV}$ conversion dynode was placed adjacent to the electron multiplier near the exit endcap electrode. Ions were formed by LD from the sample located $\sim 1.5 \mathrm{~cm}$ away from the endcap and were axially injected through the endcap into the trap, which contained $\sim 10^{-4}$ torr of helium buffer gas [6]. We used the standard mass-selective ejection scan sequence for these experiments: the radiofrequency potential on the ring electrodes was a few hundred volts during ion injection, and the sample and endcaps were always at ground potential. The timing and data collection were controlled by custom software with multifunction data acquisition bnards (National Instruments) mounted in an 80386/AT microcomputer.

The fourth harmonic output $(266 \mathrm{~nm})$ from an Nd:YAG laser (Continuum, Surelite) was focused onto the samples with power densities of $10^{6}-10^{7} \mathrm{~W} / \mathrm{cm}^{2}$. The laser power density was calculated by visual estimation of the laser spot size and measurement with a pyroelectric joulemeter (Molectron).

The commercially available gold substrates used here (Sycon Instruments) were ctched in our lab with dilute aqua regia (3:1:6 $\left.\mathrm{HCl}_{2} \mathrm{HNO}_{3}: \mathrm{H}_{2} \mathrm{O}\right)$ [10], rinsed with deionized water and absolute ethanol, then immersed overnight in $1 \mathrm{mM}$ ethanol solutions of hexanethiol (Aldrich, 96\%, Aldrich Chemical Co., Milwaukee, WI), decanethiol (Aldrich, 96\%), or hexadecanethiol (Aldrich, 92\%) to prepare the selfassembled monolayers (SAMs) [11]. The gold substrates were removed from solution the following day, rinsed with ellanol, and air dried.

After mounting a SAM sample in the vacuum chamber, the system was pumped down to a pressure 
$<6 \times 10^{-7}$ torr. The laser power was adjusted to a point just below the threshold for ablation of the gold substrate, as monitored by $\mathrm{Au}^{-}$production. After their injection into the trap, the ions were held for a few hundred milliseconds before being mass selectively ejected and detected [12]. Ion signals from 20 to 30 laser shots (scan sequences) were summed to generate a mass spectrum, where the sample was rotated to an unexposed portion of the surface for every laser shot. The summed spectra were then subjected to secondorder fast Fourier transform smoothing (Spectra Calc, Galactica Industries) to remove statistical noise.

\section{Results and Discussion}

Figure 1 shows three mass spectra displaying different alkanesulfonate negative ion peaks desorbed from the gold surfaces, depending upon the length of the polymethylene chain. Sulfonate ions with the general formula $\mathrm{CH}_{3}\left(\mathrm{CH}_{2}\right)_{n} \mathrm{SO}_{3}^{-}(\mathrm{n}=5,9$, and 15) were detected at $m / z 165,221$, and 305, respectively, from the SAMs prepared from alkanethiols of the same chain length. These sulfonate negative ions derived from the $\mathrm{CH}_{3}\left(\mathrm{CH}_{2}\right)_{\mathrm{n}} \mathrm{SO}_{3}$ adsorbate, which forms as the result of partial uxidation of tlıe alkanthiol $[2,13]$, as will be discussed below. All spectra displayed a $\mathrm{HSO}_{4}^{-}$ion peak at $m / z$ 97. No other fragments or higher mass

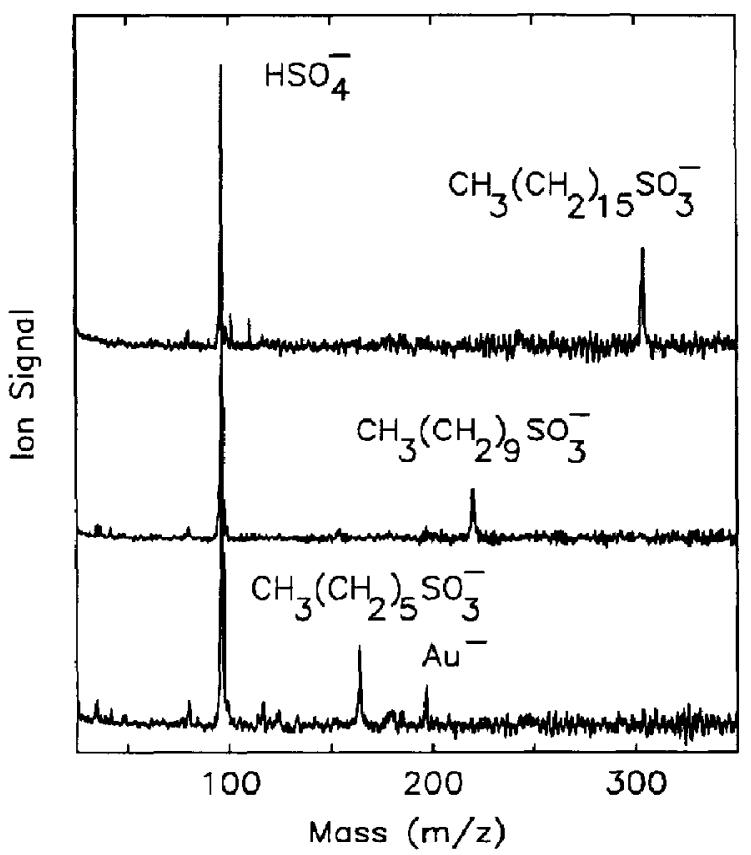

Figure 1. Negative ion mass spectra displaying alkanesulfonate ion peaks from SAMs prepared from $\mathrm{CH}_{3}\left(\mathrm{CH}_{2}\right)_{n}$ SH with $n=5$ (bottom spectrum), 9 (middle spectrum), and 15 (top spectrum) on gold. ions (up to $m / z 650$ ) were observed from any of the SAMs. Ablation of the gold surface leads to the appearance of a $\mathrm{Au}^{-}$peak at $\mathrm{m} / z 197$ in the hexanesulfonate spectrum (bottom). This ion has occasionally been observed from the other surfaces as well, depending upon the exact laser power.

Previous spectra obtained using 193 nm LD of alkanethiol monolayers into an ion cyclotron resonance mass spectrometer detected both thiolate (RS ${ }^{-}$) and sulfonate $\left(\mathrm{RSO}_{3}^{-}\right)$ions [2]. With $266 \mathrm{~nm}$ laser pulses, we were unable to detect either thiolate or sulfonate ions from freshly prepared samples, but sulfonate ions were observed in the spectra after the samples were allowed to oxidize in air for at least 6 hours before analysis. It has been estimated that this oxidation process leads to formation of a sulfonate surface concentration of less than a few percent [2]. We attribute our inability to detect the thiolate ions solely to the longer wavelength of our laser compared with that used previously. The higher sensitivity of LD for the sulfonates compared with the thiols has been attributed to the higher gas-phase acidity of sulfonic acids (for $\mathrm{RSO}_{3} \mathrm{H}$ $\left.\rightarrow \mathrm{RSO}_{3}^{-}+\mathrm{H}^{+} \Delta \mathrm{G}^{\circ} \sim 314 \mathrm{kcal} / \mathrm{mol}\right)$ than for the corresponding thiols $\left(\Delta \mathrm{G}^{\circ} \sim 350 \mathrm{kcal} / \mathrm{mol}\right)$ [2]. Our inability to detect $\mathrm{RSO}_{3}^{-}$or $\mathrm{HSO}_{4}^{-}$ions on samples which have not been oxidized in air is also in agrecment with the literature $[2,13]$ and indicates that the lack of thiolate signal is not an artifact of sample preparation.

Static secondary ion mass spectrometry (SSIMS) has also been used to analyze this adsorbate system, but the resultant spectra were dominated by fragmentations, clusters, and gold atom adducts rather than intact ions of the adsorbate $[14,15]$. The capability of LD-ITMS to delect inlacl adsorbate ions desorbed from these surfaces with little or no fragmentation, clustering, or adduct formation makes the former superior to SSIMS in terms of spectral simplicity.

Unlike SAMs, many surfaces require the use of ultrahigh vacuum techniques to prevent contamination from background gas adsorption during analysis. Given the need for a $10^{-4}$ torr pressure of helium buffer gas during analysis, parts per million impurity levels in the helium could lead to contamination of more reactive surfaces such as clean metals or semiconductors. The use of demonstrated methods of ultrapurification of the helium [16] would render LD-I'IMS suitable for the analysis of such reactive surfaces.

\section{References}

1. Lubman, D. M., Ed. Lasers and Mass Spectrometry; Oxford University Press: New York, 1990.

2. Li, Y.; Huang, J.; McIver Jr., R. T.; Hemminger, J. C. J. Am. Chem. Soc. 1992, 174, 2428-2432, and references therein.

3. March, R. E. Inter. J. Mass Spectrom. Ion Processes 1992, $118 / 119,71-135$, and references therein.

4. Cox, K. A.; Williams, J. D.; Cooks, R. G.; Kaiser Jr., R. E. Biol. Mass Spectrom. 1992, 21, 226-241. 
5. Heller, D. N.; Lys, I.; Cotter, R. J.; Uy, O. M. Anal. Chem, 1989, 61, 1083-1086.

6. McIntosh, A.; Donovan, T.; Brodbelt, J. Anal. Chem. 1992, 64, $2079-2083$.

7. Chambers, D. M.; Goeringer, D. E.; McLuckey, S. A.; Glish, G. L. Anal. Chem. 1993, 65, 14-20.

8. Jonscher, K; Currie, G.; McCormack, A. L.; Yates III, J. R. Rapid Commun. Mass Spectrom. 1993, 7, 20-26.

9. Alexander, M. L.; Hemberger, P. H.; Cisper, M. E.; Nogar, N. S. Anal. Chem. 1993, 65, 1609-1614.

10. Creager, S. E.; Hockett, L. A.; Rowe, G. K. Langmuir 1992, \&, $854-861$
11. Nuzzo, R. G.; Allara, D. L. J. Am. Chem. Soc. 1983, 105, 4481-4483.

12. Wu, H.-F; Brodbelt, J. S. Inter. I. Mass Specirom. lon Processes 1992, 115, 67-81.

13. Huang, J.; Hemminger, J. C. I. Am. Chem. Soc. 1993, 115 , 3342-3343.

14. Frisbie, C. D.; Martin, J. R.; Duff, Jr., R. R.; Wrighton, M. S. J. Am. Chem. Soc. 1992, 114, 7142-7145.

15. Tarlov, M. J.; Newman, J. G. Iangmuir 1992, 8, 1398-1405.

16. Hanley, L.; Xu, Z.; Yates, Jr., J.T. Susf. Sci. Lett. 1991, 248, L265-L273, and references therein. 